\title{
Rezensionen und Berichte
}

\section{Nationale Stereotype und Diskurskonfrontationen in den deutschen, polnischen und französischen Reiseberichten und anderen schriftlichen Medien der Frühen Neuzeit ${ }^{1}$}

\author{
Włodzimierz Zientara, Liliana Lewandowska, (Hrsg.): Das Fremde \\ erfahren. Polen-Litauen, Deutschland und Frankreich \\ in der frühneuzeitlichen Reiseliteratur. Torun 2014, 314 S.
}

DOI: $10.19195 / 0435-5865.142 .23$

Der hier besprochene Anthologie-Band entstand infolge der Kooperation zwischen der Universität Saint-Quentin-en-Yvelines Versailles und der Nikolaus-Kopernikus-Universität in Toruń. Die Beitragsverfasser_innen dieses gemeinsamen Projekts vertreten dabei unterschiedliche Fachbereiche wie die Geisteswissenschaften, Geschichts- und Kulturwissenschaften. Den Inhalt der Publikation bilden historische Quellentexte in transkribierter Form, denen eine kurze Erläuterung als Kontextualisierungshilfe vorangestellt wird. Bei den präsentierten Beiträgen handelt es sich um Ego-Dokumente, die die Zeitspanne, mit einem frühesten Beispiel aus dem 17. Jahrhundert bis zum 19. Jahrhundert, abdecken. Der Mitherausgeber Włodzimierz Zientara deklariert im Vorwort die primäre Absicht, die Publikation als Zitaten-Thesaurus zu didaktischen Zwecken an Hochschulen zu verwenden. Als Leiter der Fakultät für Ältere Literatur und Kultur gehören zu Zientaras Forschungsschwerpunkten u.a. die deutsch-polnische Beziehung, subkategorisch ebenso die nationalstereotypen Darstellungen Polens und Deutschlands in der Literatur. Die Herausgeber möchten mit dieser Anthologie den akademischen Diskurs in diesem interkulturellen Forschungsbereich aufrechterhalten.

Der gattungsspezifisch gemischte Korpus wurde nach dem Kriterium der Textfunktionalität in zwei Segmente aufgeteilt: 1) Reiseberichte; 2) Polen in unterschiedlichen Medien.

Den ersten Teil der Anthologie eröffnet Joanna Kodzik mit zwei Texten aus dem frühen 18. Jahrhundert, die die Reiseorganisation der Eliten zu Zeiten der Aufklärung darstellen. Es handelt sich im ersten Fall um das neunte Kapitel aus dem Buch „Einleitung zur Ceremoniel-Wissenschaft, Der grossen Herren“ (1733) von Julius Bernhard von Rohr (1688-1742), der sich als Zeremoniellwissenschaftler in die Geschichte eingeschrieben hat. Der zweite Textauszug hingegen entstammt dem 1755 erschienenen Werk, ,Teutsches Hofrecht" aus der Feder des Rechtsgelehrten Friedrich Carl von Moser (1723-1798). In jenem Fragment unternimmt Moser den Vergleich zwischen alten und neuen Reisepraxen in Hinblick auf den logistischen Aufwand und definiert zusätzlich verschiedene Reisearten, die von den Intentionen des Reisenden abhängig sind. Den Übergang vom deutschen zum fran-

\footnotetext{
${ }^{1}$ Zientara, Włodzimierz / Lewandowska, Liliana (Hrsg.): Das Fremde erfahren. Polen-Litauen, Deutschland und Frankreich in der frühneuzeitlichen Reiseliteratur. Torun 2014, 314 S.
} 
zösischen und italienischen Boden vollzieht Katarzyna Szczerbowska-Prusevicius, die sich in ausführlicher Weise den musikalischen Reisebeschreibungen des englischen Musikgelehrten Charles Burney (1726-1814) widmet. Im Fokus seiner Tagebucheinträge erscheinen die verschiedenartigen Musikstile Frankreichs und Italiens im komparativen Diskurs, den der Autor aus einer englisch-nationalstolzen Position führt. Im nächsten Beitrag von Maja Baran, präsentiert die Autorin einen französischsprachigen Bericht u.d.T. „Voyage de la Prusse à Warsovie“" (1780), der die Reise des preußischen Grafen Carl Ludwig Friedrich Bogislaw von Dönhoff (1754-1809) nach Warschau dokumentiert. Sein Augenmerk legte er auf die Beschreibung der polnischen Städte und Bewohner, des Adels, aber auch auf die Eigenarten der polnischen Politik am Beispiel einer Parlamentssitzung. Die Beschreibung Polens nimmt auch einen zentralen Platz in dem von Włodzimierz Zientara angeführten Text „Bemerkungen auf einer Reise von Thorn durch Posen nach Sachsen“ des Thorner Juristen Johann Christoph Hornuff (1747-1799) ein. Jener Autor der im Jahre 1790 gedruckten Schrift, zeichnet sich durch ein faires und rationales Urteilsvermögen aus, das die Reproduktion von polnischen Vorurteilen unterbindet. Gleich ein Jahr darauf wurden Johann Friedrich Zöllners (1753-1804) Briefe über seine Reise von Berlin nach Krakau veröffentlicht. Katarzyna Chlewicka entschied sich aus diesem zumal unterhaltsamen, doch ebenso informativen Reisebericht den Textauszug über das polnische Krakau abzudrucken. Die zweite Beschreibung Warschaus wird von Liliana Lewandowska im Text des Schriftstellers Joachim Christoph Friedrich Schulz (1762-1798) geliefert. In der „Reise eines Liefländers von Riga nach Warschau ..." (1795) wird die Stadt zu den Regierungszeiten Stanisław August Poniatowskis gezeigt, in der die Popularisierung der Wissenschaften ihren Aufschwung erlebte, diese jedoch von Schulz größtenteils kritisch bewertet wurde. In den Reisebeschreibungen von Karl Ephraim Nanke, herausgegeben von Ludwig van Baczko (1756-1823), wird der Anthologie-Band erstmals durch den auf Litauen bezogenen Text „Reise durch ein Theil Preussens von Ludwig von Baczko" (1800) bereichert. Mit dem Auge eines Historikers, der auch für die aktuelle Geschichtsforschung wertvolle Fakten vermittelt, beschrieb er ein wohlwollendes Bild von Litauen. Jan Born steuert einen weiteren Beitrag zur Darstellung Warschaus u.d.T. „Wilanów im Jahre 1842 aus französischer Sicht. Xavier Marmier" bei. Die Beschreibung des Schlosses und seiner Entourage verbindet der Schriftsteller Xavier Marmier (1808-1892) mit einer historischen Kontrastierung der einstigen Größe Polens mit dem zu seiner Zeit aktuellen Sklavenstatus.

Mit der „Berichtserstattung über Polen in deutschsprachigen Meßrelationen“ beginnt Esther-Beate Körber den zweiten Teil des Sammelbandes, der das konstruierte Bild Polens in unterschiedlichen Medien behandelt. Die Autorin geht kurz und prägnant auf die Geschichte dieses Informationsmediums ein, dessen Genese bis in das 16. Jahrhundert zurückreicht. Der politisch internationale Charakter der Relationen wird anhand zweier Frankfurter Beispiele aus dem 17. und 18. Jhdt. aufgezeigt, die in ihrer Polen-Darstellung durch das subjektive Eingreifen der Kompilatoren differieren. Ein dezidiert praxisorientiertes Medium wählt Marta Sikorska mit dem in Thorn herausgegeben Konversationsbuch „Polnischer Donat" von 1689. Diese deutsch-polnischen Sprechübungen des auch in Schlesien tätigen Autors Johannes Ernesti (1632-1709) basieren auf alltagsbezogenen Dialogen, die sich u.a. auf die Essenskultur der Breslauer Bürgerschicht beziehen. Warschau rückt erneut in den Fokus von Hans-Wolf Jäger, der ein Romanfragment aus dem Werk „Das galante Sachsen“ (1735) von Freiherr Karl Ludwig von Pöllnitz (1692-1775) anbietet. Das Polen-Bild beschränkt sich in diesem Beispiel auf die Figur des Königs Friedrich August II. (1696-1763) und das frivole Treiben der Warschauer Aristokratie. Der kurze Beitrag von Michael Nagel 
lenkt wieder den Diskurs bzgl. der politischen Situation Polens gegen Ende des 18. Jhdts. ein. Der dargebotene Lehrer-Schüler-Dialog aus dem Leipziger Wochenblatt für Kinder von 1772 veranschaulicht das pädagogische Ziel, die politisch komplexe Narration der Teilung Polens im simplen Kausalitätszusammenhang Kindern nahe zu bringen. Nahtlos knüpft Holger Böning an das politische Themenkomplex mit seinem Beitrag „Wie lässt sich die Bildung einer Nation auf die andere Übertragung“ an. Carl Friedrich August Grasshoff (1770-1841), Autor der 1796 erschienenen Originalschrift, verkörpert zum einen die preuBische Suprematie gegenüber der polnischen Bevölkerung, zum anderen die Vision von drastischen Bildungsreformen für den preußisch okkupierten Teil Polens.

In den Reiseberichten der Frühen Neuzeit kommt die „narrative Identität“2 der Autoren, die in den Grenzen ihres sozialen Milieus agierten, zum Vorschein. Die kulturellen und konfessionellen Grenzüberschreitungen, die sie auf ihren Reisen erfuhren, konfrontierten stets ihre Identitätskonstruktion. Folglich bietet dieser Anthologie-Band gemäß der Zielsetzung der Herausgeber, mit seiner Gattungsvielfalt und der interdisziplinären Herangehensweise an die Reiseberichtserstattung ein solides Instrumentarium für Dozenten in ihren akademischen Kursen an. Die optimale Varianz der unterschiedlichen Forschungsperspektiven für einen Sammelband wie diesen scheint nahezu erfüllt, demnach erscheinen Beiträge aus dem Bereich der Musikgeschichte, Literatur- und Kulturwissenschaft. Jene Diversität lässt sich jedoch auf der geopolitischen Ebene missen, da sich die dargebotenen Quellentexte im ersten Teil größtenteils auf Reisebeschreibung durch Polen beziehen (sechs von acht Beiträgen). Diese Quote verwundert kaum, berücksichtigt man die Tatsache, dass Polen erst in der zweiten Hälfte des 18. Jahrhunderts als Reiseziel an Popularität gewann und man mit diesem Band einer Sichtbarmachung der unbekannten Quellentexte entgegen kommen wollte. ${ }^{3}$ Hingegen irritiert die Qualität der wissenschaftlichen Bearbeitungen jener Reisetexte, die nicht Polen thematisieren. Joanna Kodzik macht auf vier Seiten zunächst die Leser_innen mit dem Reisehabitus europäischer Adelsgeschlechter vertraut und stellt gleich darauf die Kurz-Vita ihrer Autoren dar, ohne ihre bibliographischen Quellen anzugeben. Dezidiert anders verfährt Katarzyna Szczerbowska-Prusevicius in ihrer Einleitung zu dem musikhistorischen Zeitdokument, die eine äußerst interessante und sachlich fundierte Bearbeitung zu den textimmanenten Realien mit entsprechendem Quellenverweis darstellt. Ihre Erwähnung der heute wenig erforschten Pianistin und Komponistin des 18. Jhdts. Madame Brillon dürfte sicherlich Anreiz für genderorientierte Kulturforscher_innen sein, Leben und Nachlass dieser Künstlerin zu popularisieren. Dem Originaltext fügt die Autorin ihren kritischen Kommentar bei, der historische Persönlichkeiten identifiziert und zudem musikalische Fachtermini erläutert. Ein Verfahren, dessen alle Autoren_innen sich zu Gunsten der Leserschaft bedient haben. Diese hohe Informationsdichte in den Fußnoten weist ebenfalls der Beitrag von Axel Walter auf. Die aufschlussreichen bibliographischen Zusätze motivieren eine Platzierung der Gesamtbibliographie - auf die in diesem Band verzichtet wurde - am Ende jeder Einleitung. Dieser Usus, der generell für wissenschaftliche Artikel gültig ist, scheint mir für diese Publikation gerechtfertigt, da einige Beiträge, wie bereits erwähnt, weit über ein Subsumieren der Inhalte ihrer Quellentexte hinausgehen und den Zweck einer wissenschaftlichen Kontribution erfüllen. Diese formelle Modifikation käme dem Rezeptions-Komfort zu Gute. Es scheint im gleichen Zuge bedauernswert, dass nicht alle Autoren_innen dem hohen wissenschaftli-

${ }^{2}$ Vgl. Eder, Franz X. (Hrsg.): Historische Diskursanalysen. Genealogie, Theorie, Anwendungen. Wiesbaden 2006, S. 273.

${ }^{3}$ Vgl. Struck, Bernhard: Nicht West - nicht Ost. Frankreich und Polen in der Wahrnehmung deutscher Reisender zwischen 1750-1850. Göttingen 2006, S. 19. 
chen Standard der Gesamtleistung gerecht wurden. Michael Nagel, der im zweiten Teil das pädagogisch fingierte Gespräch des Lehrers mit den Kleinkindern Carlchen und Carolinchen über die Teilung Polens anführt, verzichtet auf eine kontextuelle Vertiefung und bietet stattdessen den Leser innen in einer Fußnote sieben Quellen zur autodidaktischen Lektüre an. Unter Berücksichtigung der Herausgeber-Intentionen, erscheint, wenn nicht direkt kontrovers, so dann fragwürdig, der Beitrag von Hans Wolf Jäger u.d.T. „Polen in einem Bestseller des 18. Jahrhunderts." Ungeachtet der lockeren Handhabung der Quellenangaben, erscheint zusätzlich der Publikationsgrund der „Chronique Scandaleuse“ (S. 277) von Pöllnitz in dieser Anthologie unklar. Eher im Stile einer Buchempfehlung verfasst, resümiert Jäger die von Pöllnitz klatschhaften amourösen Episoden des sächsischen Kurfürsten und Königs von Polen August II. Der Großteil der gewählten Textauszüge ist auf die Beschreibung adeliger Frauen gemünzt, die Pöllnitz in misogyner Tradition oberflächlich als stolze (Madame von Esterle), überempfindsame (Fürstin Lubomirsky) oder laszive (Madame von Wartenberg) Wesen präsentiert. Zweifelsohne erfüllt dieser Beitrag das Postulat, welches im Vorwort von Włodzimierz Zientara formuliert wurde: „Unser allgemeines Ziel ist aber, an mehrere Episoden aus der gemeinsamen Geschichte, an Stereotype, Vorurteile zu erinnern [...] und Diskussionen anzuregen." (S. 7).

Trotz geringer Mangelerscheinungen in der Bearbeitung der Quellentexte stellt „Das Fremde erfahren“ in Hinblick auf den aktuellen Diskurs über „Die Grenze als Raum, Erfahrung und Konstruktion" "4 einen wichtigen Beitrag dar, der die Nation als Konstrukt stets hinterfragt und den dynamischen Charakter von Grenzen betont. Es bleibt zu hoffen, dass dieses internationale Projekt mit der Herausgabe eines wertvollen Anthologie-Bandes wie diesem, eine Inspiration für weitere interdisziplinäre Forschungen bzgl. der subjektiven Repräsentation von (fremden) Ländern und Kulturen in diversen Medien darstellt.

\section{Literatur}

Duhamelle, Christophe / Kossert, Andreas / Struck, Bernhard (Hrsg.) (2007): Grenzregionen. Ein europäischer Vergleich vom 18. bis zum 20. Jahrhundert. Frankfurt/New York.

Eder, Franz X. (Hrsg.) (2006): Historische Diskursanalysen. Genealogie, Theorie, Anwendungen. Wiesbaden.

Struck, Bernhard (2006): Nicht West - nicht Ost. Frankreich und Polen in der Wahrnehmung deutscher Reisender zwischen 1750-1850. Göttingen.

Nicolas Antochewicz

Nicolas Antochewicz

Uniwersytet Wrocławski

Instytut Filologii Germańskiej

Pl. Biskupa Nankiera 15 b

50-140 Wrocław

Polen

E-Mail: nicolas.antochewicz@gmail.com

${ }^{4}$ Duhamelle, Christophe / Kossert, Andreas / Struck, Bernhard (Hrsg.): Grenzregionen. Ein europäischer Vergleich vom 18. bis zum 20. Jahrhundert. Frankfurt/New York 2007, S. 9. 